\title{
Derivative Dispersion Relations Above the Physical Threshold
}

\author{
R. F. Ávila \\ Instituto de Matemática, Estatística e Computação Científica \\ Universidade Estadual de Campinas, UNICAMP \\ 13083-970 Campinas, SP, Brazil \\ and M. J. Menon \\ Instituto de Física Gleb Wataghin \\ Universidade Estadual de Campinas, UNICAMP \\ 13083-970 Campinas, SP, Brazil
}

Received on 28 November, 2006

\begin{abstract}
We discuss some formal and practical aspects related to the replacement of Integral Dispersion Relations (IDR) by derivative forms, without high-energy approximations. We first demonstrate that, for a class of functions with physical interest as forward scattering amplitudes, this replacement can be analytically performed, leading to novel Extended Derivative Dispersion Relations (EDDR), which, in principle, are valid for any energy above the physical threshold. We then verify the equivalence between the IDR and EDDR by means of a popular parametrization for total cross sections from proton-proton and antiproton-proton scattering and compare the results with those obtained through other representations for the derivative relations. Critical aspects on the limitations of the whole analysis, from both formal and practical points of view, are also discussed in some detail.
\end{abstract}

Keywords: Elastic hadron scattering; Dispersion relations; High energies

\section{INTRODUCTION}

Elastic hadron scattering constitutes a hard challenge for QCD. The problem concerns the large distances involved (confinement), which renders difficult the development of a formal nonperturbative calculational scheme for scattering states, able to describe soft diffractive processes. At this stage Analyticity, Unitarity, Crossing and their consequences, still represent a fundamental framework for the development of theoretical ideas, aimed to reach efficient descriptions of the experimental data involved. In this context, dispersion relations, connecting real and imaginary parts of the scattering amplitude, play an important role as a useful mathematical tool, in the simultaneous investigation of particle-particle and antiparticle-particle scattering.

Dispersion relations in integral form, for hadronic amplitudes, were introduced in the sixties, as consequences of the Cauchy's theorem and the analytic properties of the scattering amplitude, dictated by unitarity [1-3]. However, two kinds of limitations characterize this integral approach: (1) its nonlocal character (in order to evaluate the real part, the imaginary part must be known in all the integration space); (2) the restricted class of functions that allows analytical integration. Later on it was shown that, for hadronic forward elastic scattering in the region of high and asymptotic energies, these integral relations can be replaced by derivative forms [4-6]. Since then, the formal replacement of integral by derivative relations and their practical use have been widely discussed in the literature [7-13], mainly in the seminal papers by Kolár and Fischer $[12,13]$. See Ref. [14] for a recent critical review on the subject.

Despite the results that have been obtained with the derivative approach, the high-energy condition (specifically, centerof-mass energies above $10-20 \mathrm{GeV}$ ) turns out difficult any attempt to perform global fits to the experimental data connecting information from low and high energy regions. A first step in this direction appears in Ref. [15], where new representations for the derivative relations, extended to low energies, have been introduced by Cudell, Martynov and Selyugin and to which we shall refer in what follows. However, a rigorous formal extension of the derivative dispersion relations down to the physical threshold, providing a complete analytical equivalence between integral and differential approaches, is still missing and that is the point we are interested in.

In this work, we first demonstrate that, for a class of functions of physical interest as forward elastic scattering amplitudes, the integral relations can be analytically replaced by derivative forms without the high-energy approximation. Therefore, in principle, for this class of functions, derivative relations hold for any energy above the physical threshold. We then check the consistences of the results obtained with the integral relations and the extended derivative dispersion relations by means of a simple analytical parametrization for the total cross sections from proton-proton $(p p)$ and antiprotonproton $(\bar{p} p)$ scattering (highest energy interval with available data). In addition, we compare the results with those obtained through the standard derivative relations (high-energy condition) and the derivative representation by Cudell-MatynovSelyugin. We shall show that, above the physical threshold, only the extended relations lead to exactly the same results as those obtained with integral forms. We proceed with a critical discussion on the limitations of our analysis from both formal and practical points of view.

The manuscript is organized as follows. In Sec. II we recall the main formulas and some conditions involving the Integral Dispersion Relations (IDR), the standard Derivative Dispersion Relations (sDDR) and the Cudell-Martynov-Selyugin representations (CMSr); we also present, in certain detail, the 
replacement of IDR by the Extended Derivative Dispersion Relations (EDDR). In Sec. III we check the consistences and exemplify the applicability of all these results in simultaneous fits to the total cross section and the ratio $\rho$ of the real to imaginary parts of the forward amplitude, from $p p$ and $\bar{p} p$ scattering. In Sec. IV we present a critical discussion on all the obtained results. The conclusions and some final remarks are the contents of Sec. V.

\section{DISPERSION RELATIONS}

\section{A. Integral Dispersion Relations (IDR)}

First, it is important to recall that Analyticity, Unitarity and Crossing lead to IDR for the scattering amplitudes in terms of a crossing symmetric variable. For an elastic process, $m+m \rightarrow m+m$, in the forward direction, this variable corresponds to the energy of the incident particle in the laboratory system, $E[1]$. In this context and taking into account polynomial boundedness, the one subtracted IDR for crossing even $(+)$ and odd $(-)$ amplitudes, in the physical region $(E: m \rightarrow \infty)$, read [1-3]

$$
\begin{gathered}
\operatorname{Re} F_{+}(E)=K+\frac{2 E^{2}}{\pi} P \int_{m}^{+\infty} \mathrm{d} E^{\prime} \frac{1}{E^{\prime}\left(E^{\prime 2}-E^{2}\right)} \operatorname{Im} F_{+}\left(E^{\prime}\right), \\
\operatorname{Re} F_{-}(E)=\frac{2 E}{\pi} P \int_{m}^{+\infty} \mathrm{d} E^{\prime} \frac{1}{\left(E^{\prime 2}-E^{2}\right)} \operatorname{Im} F_{-}\left(E^{\prime}\right),
\end{gathered}
$$

where $K$ is the subtraction constant.

The connections with the hadronic amplitudes for crossed channels, such as $p p$ and $\bar{p} p$ elastic scattering, are given by the usual definitions:

$$
F_{p p}=F_{+}+F_{-} \quad F_{\bar{p} p}=F_{+}-F_{-} .
$$

The main practical use of the IDR concerns simultaneous investigations on the total cross section (Optical Theorem) and the ratio $\rho$ of the real to imaginary parts of the forward amplitude, which is also our interest here. In terms of the crossing symmetric variable $E$ these physical quantities are given, respectively, by [3]

$$
\begin{gathered}
\sigma_{\mathrm{tot}}=\frac{4 \pi}{\sqrt{E^{2}-m^{2}}} \operatorname{Im} F\left(E, \theta_{\mathrm{lab}}=0\right), \\
\rho(E)=\frac{\operatorname{Re} F\left(E, \theta_{\mathrm{lab}}=0\right)}{\operatorname{Im} F\left(E, \theta_{\mathrm{lab}}=0\right)},
\end{gathered}
$$

where $\theta_{\text {lab }}$ is the scattering angle in the laboratory system.

\section{B. Standard Derivative Dispersion Relations (sDDR)}

Basically, at high energies, the replacement of IDR by sDDR is analytically performed by considering the limit $m \rightarrow$ 0 in Eqs. (1) and (2) [5, 14]. It should be recalled that an additional high-energy approximation is considered in these integral equations, when they are expressed in terms of the centerof-mass energy squared $s=2\left(m^{2}+m E\right)$ and not $E$ [5]. However, based on a rigorous replacement (discussed in Sec. II D), we consider the derivative relations in terms of the crossing symmetric variable $E$. In this case the sDDR read $[5,12,14]$

$$
\begin{gathered}
\operatorname{Re} F_{+}(E)=K+E \tan \left[\frac{\pi}{2} \frac{\mathrm{d}}{\mathrm{d} \ln E}\right] \frac{\operatorname{Im} F_{+}(E)}{E}, \\
\operatorname{Re} F_{-}(E)=\tan \left[\frac{\pi}{2} \frac{\mathrm{d}}{\mathrm{d} \ln E}\right] \operatorname{Im} F_{-}(E) .
\end{gathered}
$$

Necessary and sufficient conditions for the convergence of the above tangent series have been established by Kolár and Fischer, in particular through the following theorem [12]:

Theorem 1 Let $f: R^{1} \rightarrow R^{1}$. The series

$$
\tan \left[\frac{\pi}{2} \frac{\mathrm{d}}{\mathrm{d} x}\right] f(x)
$$

converges at a point $x \in R^{1}$ if and only if the series

$$
\sum_{n=o}^{\infty} f^{(2 n+1)}(x)
$$

is convergent.

For example, in the case of $f(x)=e^{\gamma x}, \gamma$ a real constant, the ratio test demands $|\gamma|<1$ for the series to be absolutely convergent (which will be our interest in Sec. III).

\section{Cudell-Martynov-Selyugin Representations (CMSr)}

Recently, the following representations have been introduced for the derivative dispersion relations [15]

$$
\begin{aligned}
\operatorname{Re} F_{+}(E)= & K+E \tan \left[\frac{\pi}{2} \frac{\mathrm{d}}{\mathrm{d} \ln E}\right] \frac{\operatorname{Im} F_{+}(E)}{E} \\
& -\frac{2}{\pi} \sum_{p=0}^{\infty} \frac{C_{+}(p)}{2 p+1}\left(\frac{m}{E}\right)^{2 p}
\end{aligned}
$$

where,

$$
C_{+}(p)=\frac{\mathrm{e}^{-\xi D_{\xi}}}{2 p+1+D_{\xi}}\left[\operatorname{Im} F_{+}(E)-E \operatorname{Im} F_{+}^{\prime}(E)\right] .
$$

and 


$$
\begin{aligned}
\operatorname{Re} F_{-}(E) & =-E \cot \left[\frac{\pi}{2} \frac{\mathrm{d}}{\mathrm{d} \ln E}\right] \frac{\operatorname{Im} F_{-}(E)}{E} \\
& -\frac{2}{\pi} \sum_{p=0}^{\infty} \frac{C_{-}(p)}{2 p+1}\left(\frac{m}{E}\right)^{2 p+1}
\end{aligned}
$$

where

$$
C_{-}(p)=\frac{\mathrm{e}^{-\xi D_{\xi}}}{2 p+D_{\xi}}\left[\operatorname{Im} F_{+}^{\prime}(E)\right]
$$

and $\xi=\ln (E / m)$ and $D_{\xi}=\frac{\mathrm{d}}{\mathrm{d} \xi}$.

We note the presence of correction terms in the form of infinity series, which go to zero as the energy increases, leading to the sDDR, Eqs. (6-7). We shall use this representation in Sec. III, where their applicability is discussed in detail.

\section{Extended Derivative Dispersion Relations (EDDR)}

In this section we present our analytical replacement of the IDR by derivative forms without the high-energy approximation. We also specify the class of functions for which this replacement can be formally performed.

Let us consider the even amplitude, Eq. (1). Integrating by parts we obtain

$$
\begin{aligned}
\operatorname{Re} F_{+}(E)= & K-\frac{E}{\pi} \ln \left|\frac{m-E}{m+E}\right| \frac{\operatorname{Im} F_{+}(m)}{m} \\
& -\frac{E}{\pi} \int_{m}^{\infty} \ln \left|\frac{E^{\prime}-E}{E^{\prime}+E}\right| \frac{\mathrm{d}}{\mathrm{d} E^{\prime}} \frac{\operatorname{Im} F_{+}\left(E^{\prime}\right)}{E^{\prime}} \mathrm{d} E^{\prime} .
\end{aligned}
$$

Following Ref. [15], we define $E^{\prime}=m \mathrm{e}^{\xi^{\prime}}$ and $E=m \mathrm{e}^{\xi}$, so that the integral term in the above formula is expressed by

$$
\frac{m \mathrm{e}^{\xi}}{\pi} \int_{m}^{\infty} \ln \operatorname{coth}\left(\frac{1}{2}\left|\xi^{\prime}-\xi\right|\right) \frac{\mathrm{d}}{\mathrm{d} \xi^{\prime}} g\left(\xi^{\prime}\right) \mathrm{d} \xi^{\prime},
$$

where $g\left(\xi^{\prime}\right)=\operatorname{Im} F\left(m \mathrm{e}^{\xi^{\prime}}\right) /\left(m \mathrm{e}^{\xi^{\prime}}\right)$. Expanding the logarithm in the integrand in powers of $x=\xi^{\prime}-\xi$,

$$
\ln \left(\cot \frac{1}{2}|x|\right)=\ln \left(\frac{1+\mathrm{e}^{-|x|}}{1-e^{-|x|}}\right)=2 \sum_{p=0}^{\infty} \frac{\mathrm{e}^{-(2 p+1)|x|}}{2 p+1}
$$

and assuming that

$$
\frac{\mathrm{d}}{\mathrm{d} \xi^{\prime}} g\left(\xi^{\prime}\right) \equiv \tilde{g}\left(\xi^{\prime}\right)
$$

is an analytic function of its argument, we perform the expansion

$$
\begin{aligned}
\tilde{g}\left(\xi^{\prime}\right) & =\left.\sum_{n=0}^{\infty} \frac{\mathrm{d}^{n}}{\mathrm{~d} \xi^{\prime n}} \tilde{g}\left(\xi^{\prime}\right)\right|_{\xi^{\prime}=\xi} \frac{\left(\xi^{\prime}-\xi\right)^{n}}{n !} \\
& =\sum_{n=0}^{\infty} \frac{\tilde{g}^{(n)}(\xi)}{n !}\left(\xi^{\prime}-\xi\right)^{n} .
\end{aligned}
$$

Substituting the above formulas in Eq. (11) and integrating term by term, under the assumption of uniform convergence of the $\tilde{g}\left(\xi^{\prime}\right)$ series, we obtain

$$
\frac{2 m \mathrm{e}^{\xi}}{\pi} \sum_{p=0}^{\infty} \frac{1}{2 p+1} \sum_{k=0}^{\infty} \frac{1}{k !} \frac{\mathrm{d}^{k}}{\mathrm{~d} \xi^{k}} \tilde{g}(\xi) I_{k p}
$$

where

$$
I_{k p}=\int_{0}^{\infty} \mathrm{e}^{-(2 p+1)\left|\xi^{\prime}-\xi\right|}\left(\xi^{\prime}-\xi\right)^{k} \mathrm{~d} \xi^{\prime}=\frac{1}{(2 p+1)^{k+1}}\left[\left((-1)^{k}+1\right) k !-(-1)^{k} \Gamma(k+1,(2 p+1) \xi)\right]
$$

and $\Gamma$ is the incomplete gamma function $\Gamma(a, z)=$ $\int_{z}^{\infty} t^{a-1} \mathrm{e}^{-t} \mathrm{~d} t$.
With this procedure and from $\xi=\ln (E / m)$, Eq. (10) is expressed by

$$
\begin{aligned}
\operatorname{Re} F_{+}(E)= & K-\frac{E}{\pi} \ln \left|\frac{m-E}{m+E}\right| \frac{\operatorname{Im} F_{+}(m)}{m}+\frac{4 E}{\pi} \sum_{p=0}^{\infty} \sum_{k=0}^{\infty} \frac{1}{(2 p+1)^{2 k+2}} \frac{\mathrm{d}^{2 k+1}}{\mathrm{~d}(\ln E)^{2 k+1}} \frac{\operatorname{Im} F_{+}(E)}{E} \\
& +\frac{2 E}{\pi} \sum_{k=0}^{\infty} \sum_{p=0}^{\infty} \frac{(-1)^{k+1} \Gamma(k+1,(2 p+1) \xi)}{(2 p+1)^{k+2} k !} \frac{\mathrm{d}^{k+1}}{\mathrm{~d}(\ln E)^{k+1}} \frac{\operatorname{Im} F_{+}(E)}{E}
\end{aligned}
$$

which can be put in the final form 


$$
\operatorname{Re} F_{+}(E)=K+E \tan \left(\frac{\pi}{2} \frac{\mathrm{d}}{\mathrm{d} \ln E}\right) \frac{\operatorname{Im} F_{+}(E)}{E}+\Delta^{+}(E, m)
$$

where the correction term $\Delta^{+}$is given by

$$
\Delta^{+}(E, m)=-\frac{E}{\pi} \ln \left|\frac{m-E}{m+E}\right| \frac{\operatorname{Im} F_{+}(m)}{m}+\frac{2 E}{\pi} \sum_{k=0}^{\infty} \sum_{p=0}^{\infty} \frac{(-1)^{k+1} \Gamma(k+1,(2 p+1) \ln (E / m))}{(2 p+1)^{k+2} k !} \frac{\mathrm{d}^{k+1}}{\mathrm{~d}(\ln E)^{k+1}} \frac{\operatorname{Im} F_{+}(E)}{E} .
$$

With analogous procedure for the odd relation we obtain

$$
\operatorname{Re} F_{-}(E)=\tan \left(\frac{\pi}{2} \frac{\mathrm{d}}{\mathrm{d} \ln E}\right) \operatorname{Im} F_{-}(E)+\Delta^{-}(E, m)
$$

where

$$
\Delta^{-}(E, m)=-\frac{1}{\pi} \ln \left|\frac{m-E}{m+E}\right| \operatorname{Im} F_{-}(m)+\frac{2}{\pi} \sum_{k=0}^{\infty} \sum_{p=0}^{\infty} \frac{(-1)^{k+1} \Gamma(k+1,(2 p+1) \ln (E / m))}{(2 p+1)^{k+2} k !} \frac{\mathrm{d}^{k+1}}{\mathrm{~d}(\ln E)^{k+1}} \operatorname{Im} F_{-}(E) .
$$

Equations (12) and (13) are the novel EDDR, which are valid, in principle, for any energy above the physical threshold, $E=m$.

We note that the correction terms $\Delta^{ \pm} \rightarrow 0$ as $E \rightarrow \infty$, leading, in this case, to the sDDR, Eqs. (6) and (7). We also note that the structure of the CMSr, Eqs. (8) and (9), are similar to the above results, but without the logarithm terms. These terms come from the evaluation of the primitive at the lower limit in the integration by parts.

Since Theorem 1 insures the uniform convergence of the series expansion associated with

$$
\tilde{g}=\frac{\mathrm{d}}{\mathrm{d} \ln E} \frac{\operatorname{Im} F(E)}{E}
$$

the condition imposed by this Theorem defines the class of functions for which the EDDR hold. For example, that is the case for $f(x)=e^{\gamma x}, 0<\gamma<1$, referred to in Sec. II B. Other conditions are discussed by Kolár and Fischer [12].

\section{PRACTICAL EQUIVALENCES BETWEEN THE INTEGRAL AND THE DERIVATIVE APPROACHES}

In this section we verify and discuss the consistences between the analytical structures of the IDR and the EDDR in a specific example: the connections of the total cross section with the $\rho$ parameter from $p p$ and $\bar{p} p$ scattering. Firstly, it is important to note that the efficiency of both integral and derivative approaches in the description of the experimental data, depends, of course, on the theory available, namely the input for the imaginary part of the amplitude. In the absence of a complete model, valid for any energy above the physical threshold, we shall consider only as a framework, a PomeronReggeon parametrization for the scattering amplitude [16, 17].
For $p p$ and $\bar{p} p$ scattering this analytical model assumes nondegenerate contributions from the even $(+)$ and odd $(-)$ secondary reggeons $\left(a_{2} / f_{2}\right.$ and $\rho / \omega$, respectively), together with a simple pole Pomeron contribution:

$$
\operatorname{Im} F(E)=X E^{\alpha_{I P}(0)}+Y_{+} E^{\alpha_{+}(0)}+\tau Y_{-} E^{\alpha_{-}(0)},
$$

where $\tau=+1$ for $p p$ and $\tau=-1$ for $\bar{p} p$. As usual, the Pomeron and the even/odd reggeon intercepts are expressed by

$$
\alpha_{I P}(0)=1+\varepsilon, \quad \alpha_{+/-}(0)=1-\eta_{+/-} .
$$

We stress that the Pomeron-Reggeon phenomenology is intended for the high-energy limit (rigorously, $E$ or $\sqrt{s} \rightarrow \infty$ ). Its use here, including the region of low energies, has only a framework character. However, as we shall show, this model is sufficient for a comparative analysis of the consistences. We shall return to this aspect in Sec. IV.

In what follows, the point is to treat simultaneous fits to the total cross section and the $\rho$ parameter from $p p$ and $\bar{p} p$ scattering and compare the results obtained with both IDR and EDDR. Schematically, with parametrization (15-16) we determine $\operatorname{Im} F_{+/-}(E)$ through Eq. (3) and then $\operatorname{Re} F_{+/-}(E)$ either by means of the IDR, Eqs. (1-2) or the EDDR, Eqs. (12-13). Returning to Eq. (3) we obtain $\operatorname{Re} F_{p p}(E)$ and $\operatorname{Re} F_{\bar{p} p}(E)$ and, at last, Eqs. (4) and (5) lead to the analytical connections between $\sigma_{\text {tot }}(E)$ and $\rho(E)$ for both reactions. Moreover, through the same procedure, we shall also compare the above results with those obtained by means of both the sDDR, Eqs. (6-7) and the CMSr, Eqs. (8-9). We first present the fit procedure and then discuss all the obtained results. 


\section{A. Fitting and Results}

For the experimental data on $\sigma_{\text {tot }}(s)$ and $\rho(s)$, we made use of the Particle Data Group archives [18], to which we added the values of $\rho$ and $\sigma_{\text {tot }}$ from $\bar{p} p$ scattering at $1.8 \mathrm{TeV}$, obtained by the E811 Collaboration [19]. The statistical and systematic errors were added in quadrature. The fits were performed through the CERN-Minuit code, with the estimated errors in the free parameters corresponding to an increase of the $\chi^{2}$ by one unit. To fit the data as function of the center-of-mass energy, we express the lab energy in the corresponding formulas in terms of $s$, namely $E=\left(s-2 m^{2}\right) / 2 m$.

We included all the data above the physical threshold, $\sqrt{s}>$ $2 m \approx 1.88 \mathrm{GeV}$, that is, we did not perform any kind of data selection. Since the ensemble has a relatively large number of experimental points just above the threshold, the statistical quality of the fit is limited by the model used here as framework. In fact, with these choices and procedures we obtained reasonable statistical results (in terms of the $\chi^{2}$ per degree of freedom) only for an energy cutoff of the fits at $\sqrt{s}_{\min }=4$ $\mathrm{GeV}$. However, we stress that our focus here is in tests on the consistences among the different relations and representations and not, strictly, on the statistical quality of the fits (we shall return to this point in Sec. IV).

In each of the four cases (IDR, sDDR, CMSr and EDDR), we consider two variants of the fits, one neglecting the subtraction constant (that is, taking $K=0$ ) and the other considering the subtraction constant as a free fit parameter. The numerical results and statistical information on the fits are displayed in Table I $(K=0)$ and Table II ( $K$ free). The corresponding curves together with the experimental data are shown in Fig. $1(K=0)$ and Fig. 2 ( $K$ free $)$

\section{B. Discussion}

The main goal of this section is to discuss the consistences among the results obtained by means of distinct analytical connections between the real and imaginary parts of the amplitude. However, some phenomenological consequences can also be inferred from this study, as discussed in what follows.

From Tables I and II we see that, as expected, the best statistical results are obtained with the subtraction constant $K$ as a free fit parameter. However, as we shall show, taking $K=0$ gives suitable information not only on the practical equivalence between the IDR and the differential forms (sDDR, CMSr and EDDR), but also on the important role played by the subtraction constant. For that reason we shall treat separately the cases $K=0$ and $K$ as a fit parameter.

\section{Neglecting the subtraction constant}

From Table I we see that, for $K=0$, the numerical results obtained with the IDR and the EDDR are exactly the same, up to four figures and that this does not occur in the case with the sDDR and the CMSr neither. That is an important result since
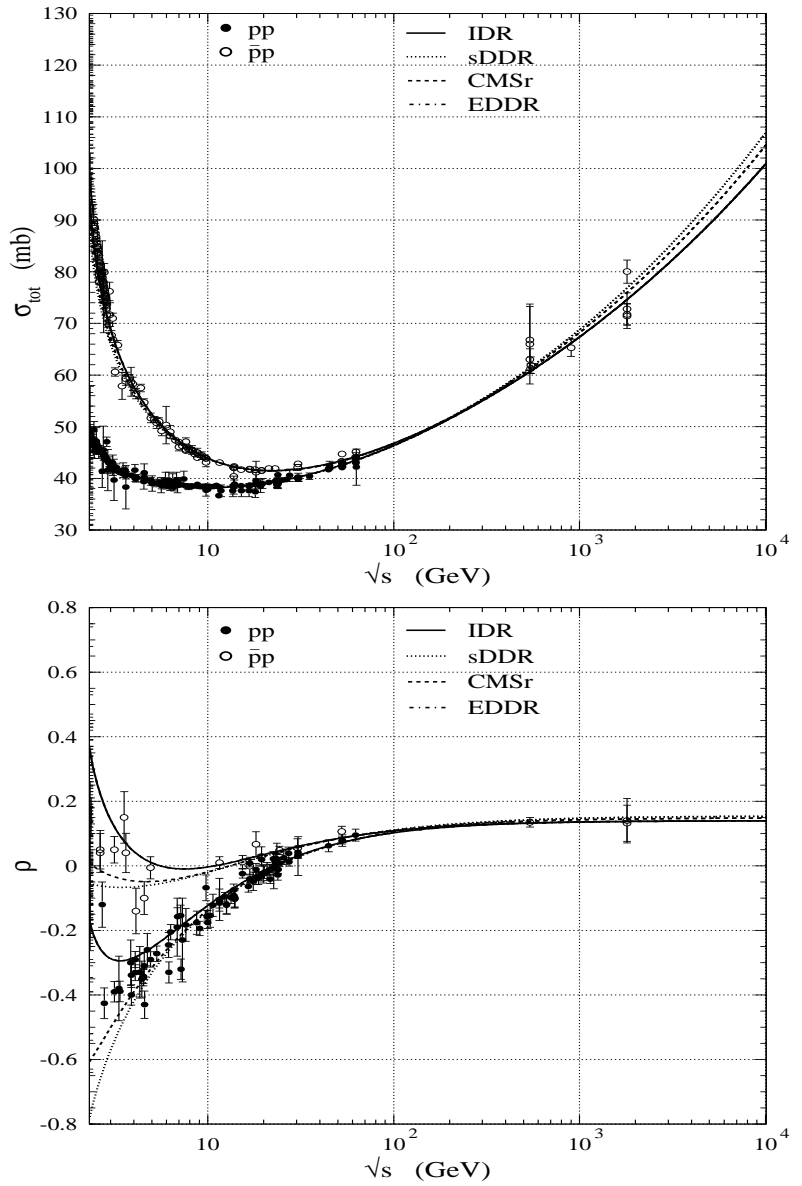

FIG. 1: Results of the simultaneous fit to $\sigma_{\text {tot }}$ and $\rho$ from $p p$ and $\bar{p} p$ scattering, by means of Integral Dispersion Relations (IDR), standard Derivative Dispersion Relations (sDDR), Cudell-Martynov-Selyugin representation (CMSr) and the Extended Derivative Dispersion Relations (EDDR) and considering the subtraction constant $K=0$ (Table I). The curves corresponding to IDR (solid) and EDDR (dot-dashed) coincide.

it demonstrates the accuracy of our analytical results for the extended derivative relations.

We note that the high values of $\chi^{2} / D O F$, in all the cases, are consequences of the specific analytical model considered (intended for the high-energy region) and the energy cutoff used. We add the fact that we did not performed any data selection, but used all the available data from the PDG archives. However, as already commented this disadvantage has no influence in our main goal, namely tests of consistences.

The effects of the equivalences (IDR and EDDR) and differences (IDR and sDDR or CMSr) in the description of the experimental data are shown in Fig. 1. The curves corresponding to IDR (solid) and EDDR (dot-dashed) coincide at all the energies above the threshold and we see that even with the fit cutoff at $\sqrt{s}_{\min }=4 \mathrm{GeV}$, the description of the experimental data below this point is reasonably good in both cases. On the other hand, the differences between the exact results (IDR and EDDR) and the sDDR or CMSr are remarkable for $\sigma_{\text {tot }}(s)$ at the highest energies and for $\rho(s)$ in the region of low 
TABLE I: Simultaneous fits to $\sigma_{\text {tot }}$ and $\rho$, from $p p$ and $\bar{p} p$ scattering, for $\sqrt{s}_{\min }=4 \mathrm{GeV}$ (270 data points), with $K=0$ and using Integral Dispersion Relations (IDR), standard Derivative Dispersion Relations (sDDR), Cudell-Martynov-Selyugin representations (CMSr) and the Extended Derivative Dispersion Relations (EDDR).

\begin{tabular}{ccccc}
\hline \hline & IDR & sDDR & CMSr & EDDR \\
\hline$X(\mathrm{mb})$ & $1.662 \pm 0.033$ & $1.497 \pm 0.032$ & $1.563 \pm 0.033$ & $1.662 \pm 0.033$ \\
$Y_{+}(\mathrm{mb})$ & $4.089 \pm 0.058$ & $3.800 \pm 0.041$ & $3.892 \pm 0.047$ & $4.089 \pm 0.058$ \\
$Y_{-}(\mathrm{mb})$ & $-2.143 \pm 0.084$ & $-1.947 \pm 0.070$ & $-2.039 \pm 0.076$ & $-2.143 \pm 0.084$ \\
$\varepsilon$ & $0.0884 \pm 0.0021$ & $0.0975 \pm 0.0021$ & $0.0939 \pm 0.0021$ & $0.0884 \pm 0.0020$ \\
$\eta_{+}$ & $0.3797 \pm 0.0099$ & $0.3209 \pm 0.0076$ & $0.3427 \pm 0.0087$ & $0.3797 \pm 0.099$ \\
$\eta_{-}$ & $0.569 \pm 0.011$ & $0.5583 \pm 0.0098$ & $0.567 \pm 0.010$ & $0.569 \pm 0.011$ \\
$\chi^{2}$ & 382.1 & 365.4 & 325.9 & 382.1 \\
$\chi^{2} /$ DOF & 1.45 & 1.38 & 1.23 & 1.45 \\
\hline \hline
\end{tabular}

TABLE II: Same as Table I but considering the subtraction constant $K$ as a free fit parameter.

\begin{tabular}{ccccc}
\hline \hline & IDR & sDDR & CMSr & EDDR \\
\hline$X(\mathrm{mb})$ & $1.598 \pm 0.034$ & $1.598 \pm 0.034$ & $1.598 \pm 0.034$ & $1.598 \pm 0.034$ \\
$Y_{+}(\mathrm{mb})$ & $3.957 \pm 0.053$ & $3.957 \pm 0.053$ & $3.957 \pm 0.053$ & $-2.084 \pm 0.080$ \\
$Y_{-}(\mathrm{mb})$ & $-2.082 \pm 0.080$ & $-2.083 \pm 0.079$ & $-2.082 \pm 0.080$ \\
$\varepsilon$ & $0.0919 \pm 0.0021$ & $0.0919 \pm 0.0021$ & $0.0919 \pm 0.0022$ & $0.0919 \pm 0.0021$ \\
$\eta_{+}$ & $0.3554 \pm 0.0098$ & $0.3555 \pm 0.0097$ & $0.3555 \pm 0.0098$ & $0.3554 \pm 0.0098$ \\
$\eta_{-}$ & $0.569 \pm 0.010$ & $0.569 \pm 0.010$ & $0.569 \pm 0.010$ & $0.569 \pm 0.010$ \\
$K$ & $-2.27 \pm 0.28$ & $2.28 \pm 0.33$ & $1.00 \pm 0.29$ & $-2.27 \pm 0.28$ \\
$\chi^{2}$ & 315.4 & 314.6 & 314.2 & 315.4 \\
$\chi^{2} /$ OF & 1.20 & 1.20 & 1.19 & 1.20 \\
\hline \hline
\end{tabular}

energies (below $\sqrt{s} \approx 10 \mathrm{GeV}$ ).

In the case of the total cross section, the results with sDDR and CMSr indicate a faster increase with the energy then those with the IDR and EDDR. We stress the importance of this point, since it gives different solutions for the well known puzzle between the CDF data [20] and the E710/E811 data $[21,22]$ at $\sqrt{s}=1.8 \mathrm{TeV}$; in this respect, we see that the exact results (IDR and EDDR) favor the E811/E710 results. In particular the values for the Pomeron intercept read (Table I): $\alpha_{I P}(0)=1.0884 \pm 0.0021$ (IDR and EDDR), $1.0975 \pm 0.0021$ $(\mathrm{sDDR})$ and $1.0939 \pm 0.0021(\mathrm{CMSr})$.

\section{Subtraction constant $K$ as a free fit parameter}

With $K$ as a free fit parameter our results demonstrate, once more, an effect that we have already noted before [14], namely the high-energy approximation can be absorbed by the subtraction constant. In fact, from Fig. 2 we see that in this case, the differences between the sDDR/CMSr and the exact results IDR/EDDR, practically disappear. From Table II we can identify the subtraction constant as the responsible for this complementary effect: the numerical values of the fit parameters and errors are practically the same in all the four cases, except for the values of $K$, that is, in practice, the differences are absorbed by this parameter. We conclude that the subtraction constant affects the fit results even in the region of the highest energies; this effect is due to the correlations among the free parameter in the fit procedure, as previously observed
$[14,23]$. Of course, also in this case the numerical values obtained with the IDR and EDDR are exactly the same, including the value of the subtraction constant up to four figures (Table II).

In particular, we note that all the four variants indicate the same result for the intercept of the Pomeron, namely $\alpha_{I P}(0)=$ $1.0919 \pm 0.0021$. The corresponding result for the total cross section lies nearly between the CDF and E811/E710 results, barely favoring the last ones (Figure 2).

\section{CRITICAL REMARKS}

We have demonstrated that for the class of functions defined by Theorem 1, IDR can be formally replaced by differential operators without any high-energy approximation; we have also verified the equivalence between the integral and extended derivative results, in the particular case of a simple phenomenological parametrization for $p p$ and $\bar{p} p$ scattering. Despite the encouraging results reached the whole analysis has limitations from both formal and practical points of view. In what follows we summarize the main critical points, giving references where more details can be found and providing also suggestions for further investigations. Since the EDDR involve two contributions, the tangent operators (SDDR) and the correction terms $\Delta^{+/-}(E, m)$, we shall consider the two cases separately. We also present some critical remarks on the Pomeron-Reggeon model used in Sect. III as a practical framework. 

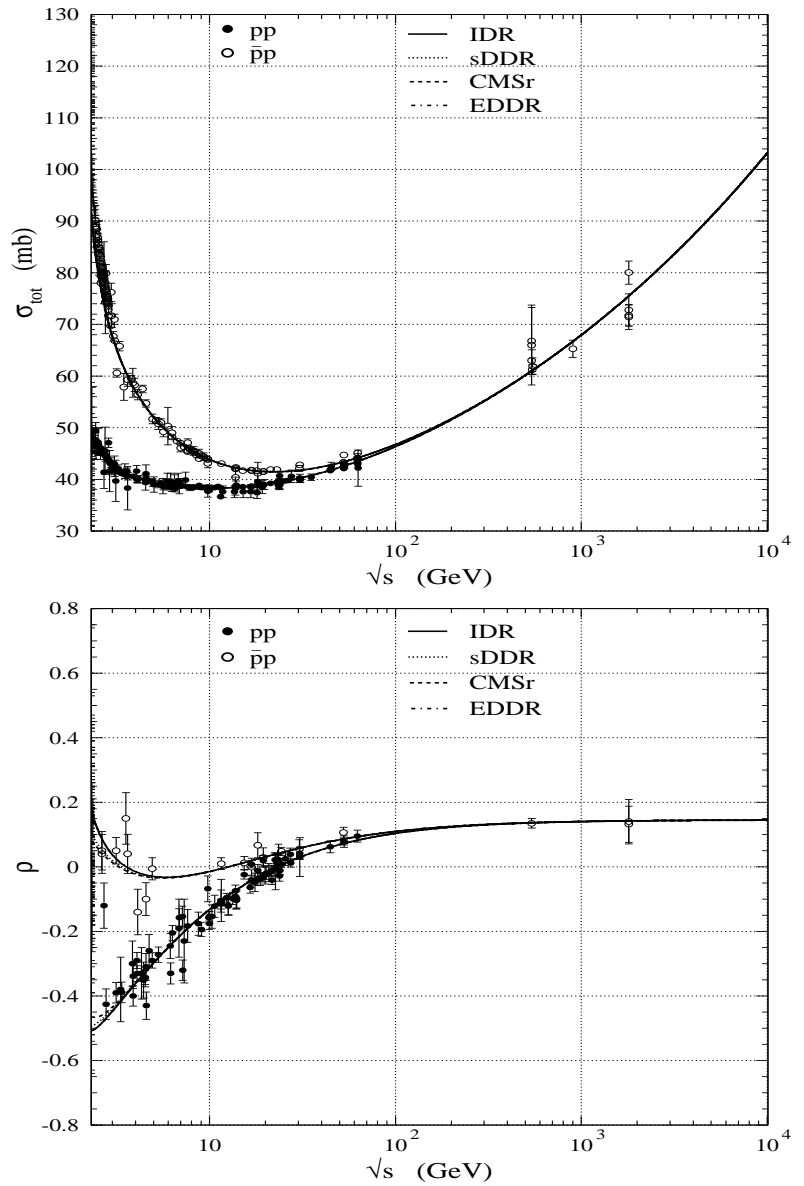

FIG. 2: Same as Figure 1 but considering the subtraction constant $K$ as a free fit parameter (Table II).

\section{A. SDDR}

First, let us discuss some aspects related to the dispersion approach as it has been treated and widely used in the literature till now, namely the sDDR, Eqs. (6) and (7). As commented in Sect. II B, these equations are obtained by considering the limit $m \rightarrow 0$ in the IDR, Eqs. (1) and (2). This condition is a critical one, which puts serious practical and formal limitations in any use of the sDDR, because that means to go to lower energies by passing through different thresholds, resonances, poles, up to $E=0$ ! In this sense, the expression of the tangent operator as an integral from $E=0$ to $E=\infty$ does not guarantee any local character for the differential approach (or the corresponding integral), even in the case of convergence of the series. In other words, this representation of the non-local operator (integral) in terms of local operators (tangent series), does not guarantee the non-locality of the result. Moreover, the representation does not apply near the resonances [7] and the convergence of the series has been discussed in several works leading some authors to argue that, in a general sense, the mathematical condition for the convergence "excludes all cases of physical interest" [7]. These and other aspects were extensively discussed in the seventies and eighties [7-13] and some points have been recently reviewed in [14].

However, there is a fundamental point developed by some authors that enlarger the practical applicability of the sDDR under some special conditions [7, 12]. As stated by Kolář and Fischer [13], in discussing the replacement of IDR by sDDR we must distinguish two formulations: (1) to consider the case of asymptotic energies and a finite number of terms in the tangent series; (2) to consider finite energies and an infinity number of terms in the series. The former case applies for smooth behaviors of the amplitude (as it is the case at sufficiently high energies, specifically $\sqrt{s}>10-20 \mathrm{GeV}$ ). That includes a wide class of functions of physical interest, mainly if only the first term can be considered [7, 12] (see [25] for a recent analysis even beyond the forward direction). The later case, however, is critical for at least two reasons. First, because the condition of convergence of the series (Theorem 1) limits the class of functions of practical applicability. Secondly and more importantly, since the high-energy approximation is enclosed, all the strong limitations referred to before applies equally well to this case. In conclusion, the class of functions for which the sDDR have a practical applicability depends strongly on the formalism considered and is narrower in the case of finite energies, namely entire functions in the logarithm of the energy.

\section{B. EDDR}

Let us now discuss the EDDR, with focus on the role of the correction terms $\Delta^{+/-}(E, m)$ in Eqs. (12) and (13). First we note that these infinity series are analytically associated with the fixed lower limit $m$ in the integral representation and they correspond to the contributions that are neglected in the highenergy approximation $(m \rightarrow 0)$. For example, for the even case we have the formal identity

$$
\begin{aligned}
& \tan \left(\frac{\pi}{2} \frac{\mathrm{d}}{\mathrm{d} \ln E}\right) \frac{\operatorname{Im} F_{+}(E)}{E}+\frac{\Delta^{+}(E, m)}{E}= \\
& \quad=\frac{2 E}{\pi} P \int_{m}^{+\infty} \mathrm{d} E^{\prime} \frac{1}{E^{\prime}\left(E^{\prime 2}-E^{2}\right)} \operatorname{Im} F_{+}\left(E^{\prime}\right),
\end{aligned}
$$

which means that all the physical situation concerns the region above the physical threshold $E=m$. Therefore, from a formal point of view, the critical points raised above on the sDDR (tangent operator only), concerning the infinity series in the region $E: 0 \rightarrow m$, do not apply in this case and the critical point here concerns only the convergence of the correction series and their practical applicability.

On the one hand, from a formal point of view (as already discussed at the end of Sect. IID), the convergence of the correction series is ensured by Theorem 1 and that means a narrower class of functions than that associated only with entire functions in the logarithm of the energy. This restriction is due to the infinity number of derivatives in $\ln s$. We shall give and discuss some examples in what follows.

From a practical point of view, it is obvious that the efficiency and/or real applicability of not only the derivative approach (EDDR), but also the integral one (IDR), depends on 
the specific physical problem involved. In this scenario (the physical problem) we expect to find some specific limitations that are independent of the formal aspects referred to above and these aspects demands also some comments. In principle and in a general sense, if we attempt to apply dispersion techniques directly to the experimental data (related to the imaginary part of a function), we are faced with the problem of error propagation from the experimental uncertainties. Even if we can "reproduce" the experimental behavior by means of suitable analytical parameterizations, with statistical errors inferred for the free parameters, these errors should, in principle, be propagated too. In this case, the infinity series in both SDDR and EDDR have certainly limited usefulness (see for example [9, 10] for the sDDR case). However, if error propagation from the fit results is not of interest or can be neglected, and, most importantly, one has a "correct" or acceptable model for the imaginary part of the amplitudes, then we are restricted only to the the formal conditions discussed above and the derivative approach becomes reliable, including the correction series (Theorem 1).

Let us now discuss the specific physical problem that motivated the present analysis. As commented in our introduction and in Sect. II, we focused the dispersion techniques in the context of hadron scattering, in special in the elastic case, for which a complet theory is still absent. The main goal concerns the connections between total cross section and the $\rho$ parameter for energies above $\sqrt{s}=5-10 \mathrm{GeV}$. In terms of dispersion techniques the usual way to treat the subject is by means of IDR, sDDR and the analyticity prescriptions for even and odd amplitudes (and recently the CMSr). In this specific case, besides the absence of a pure QCD treatment, the subject is characterized by three kinds of problems: (1) formal justification of the usual phenomenology; (2) approximated descriptions of the experimental data by phenomenological models; (3) experimental data available (problems (2) and (3) are certainly connected). Since these problems affect the practical applicability and efficiency of the dispersion techniques, let us shortly discuss some aspects involved.

(1) As it is well know, the usual phenomenology for the total cross sections is based on the reggeon concepts and involves distinct contributions from Pomerons and secondaries Reggeons. In this context, analytical parameterizations for the total cross sections are characterized by power and logarithm functions of the energy (Reggeons, simple, double and triple pole Pomerons) and the fits are performed not below $\sqrt{s}=5 \mathrm{GeV}$. We note that all these contributions belong to the class of functions defined by Theorem 1 (the tangent series can be summed leading to closed analytical results) and they have been used and investigated in several works $[15,17,23,24]$. However, as we have already pointed out [14], the central problem here concerns the fact that these contributions are formally justified only for asymptotic energies $(E$ or $s \rightarrow \infty)$, which certainly is not the case for the energies considered. The applicability of these models seems to be justified only under the hypothesis that the accelerators have already reached the energies that can be considered asymptotic in the mathematical context, which seems to us a dangerous assumption.
(2 - 3) A close look at the bulk of experimental data available shows that these ensembles present several discrepancies due to spurious data, normalization problems and other effects. In this respect, recent analysis have pointed out the necessity of some screening criterion in order to select the "correct" experimental information. We shall not discuss this question here because it seems to us an open problem. But the point is that this fact puts serious limitations in any interpretation of statistical tests of the fits, as the popular $\chi^{2}$ per degree of freedom and, consequently, not only in the efficiency of the phenomenological descriptions, but also in the possible selection of the best phenomenological model.

At last let us return to the applicability of the EDDR, now in this context. Despite of all the above problems, the known and usual phenomenological approach is characterized by analytical parameterizations for the imaginary parts of the amplitude and statistical tests on the quality of the fit. In this case, with specific analytical representations for the total cross sections, without error propagation from the fit parameters and in the Regge context we understand that the correction terms, we have introduced, can have a suitable applicability in the context of the dispersion techniques. The point is that the class of functions for which they hold includes all the usual Regge parameterizations and since the high-energy approximations is absent the fits can be formally extend to lower energies. However to reach a good statistical description of the data, specifically near the threshold, demands a "correct" model for the imaginary part of the amplitude, which, to our knowledge is still lacking. We shall return to this point in what follows.

\section{Pomeron-reggeon parametrization}

Based on all the above limitations in the phenomenological context, we have chosen one of the possible (and popular) models in order to check the equivalences (and differences) among the different dispersion representations analyzed in this work. Although, as demonstrated in Sect. III, this choice is sufficient for our aim, some drawnbacks involved demand additional comments.

In the mathematical context, as demonstrated by Kolár̆ and Fischer [12], some formal results, theorems and representations for the derivative relations were obtained under the assumption of the Froissart-Martin bound, $\sigma_{\text {tot }}<c \ln ^{2} s$, but other forms of sDDR do not require this bound. Therefore, since the simple pole Pomeron contribution, that dominates at the asymptotic energies, violates this bound, the model assumed is not an example in full agreement with the totality of the formal results. However, as already exemplified (Sect. II B), the model belongs to the class of functions defined by Theorem 1 and therefore, in this restrictive sense it seems to us to be an acceptable choice.

In the formal phenomenological context, when applied below asymptotic (infinity) energies, the model suffers from all the drawbacks already discussed. Despite of this, its use above, let us say, $\sqrt{s}=10 \mathrm{GeV}$, could be explained (not justified) by the fact that the Regge approach is the only known formalism, able to describe some global characteristics of the 
soft scattering. What is presently expected is the development of a microscopic theory able to justify its efficiency.

Now let us focus in the low energy region, above the physical threshold, $2 m_{p} \approx 1.88 \mathrm{GeV}<\sqrt{s} \leq 10 \mathrm{GeV}$ and discuss the usefullness and practical applicability of the EDDR. To our knowledge, there is no model proposed for this interval and that could explain the fact that fit procedures, even through IDR, make use of energy cutoffs at $\sqrt{s} \approx 5 \mathrm{GeV}$ ([26] is a typical example). In this sense, the usefulness of the correction terms $\Delta^{+/-}(E, m)$ could be questioned. However, we understand that the lack of a phenomenological approach for that region may also be a mirror of the present stage, characterized by a focus (probably excessive) on the highest and asymptotic energies (the great expectations from the Tevatron, RHIC, LHC). In our oppinion, independently of the fact that the "asymptopia" might be resolved or not in a short term, the connection between resonance region (above the physical threshold) and the high-energy region (above $10 \mathrm{GeV}$ ) still remains a fundamental problem demanding solution. In this respect we understand that the EDDR can play an important role in further investigations.

Concerning the practical applicability of the extended relations in this region, it is obviously limited, due to the lack of a "correct" or accepted analytical model for the imaginary part of the amplitude. One way to circumvent this problem could be the introduction of a different parametrization for this particular region. That was the procedure used in Ref. [15]; although without justification or explicit reference to the analytical form used, the authors obtained reasonable fit results. However, beyond the lack of any physical meaning, this procedure puts limitations on the equivalence between integral and derivative representations.

Based on the above facts and aimed only to check and compare the results obtained through different dispersion representations, we considered the Pomeron-Reggeon parametrization extended up to the low energy region, with a fit cutoff at $\sqrt{s}=4 \mathrm{GeV}$. Certainly the statistical results displayed in Tables I and II indicate that the confidence level is very low and even a look at Figs. 1 and 2 shows that the data near the resonance are not adequately described. As a consequence the numerical results in Tables I and II may be questionable on physical grounds. However, we insist that all the figures in these Tables are fundamental for a definite check of all the analytical representations investigated (which is the only aim of Sect. III). At last we note that one may think that it might be possible to find a suitable function, in agreement with the convergence condition and able to fit all the experimental data of interest on secure statistical grounds; that would be enough for our tests of consistences. We are not sure about this possibility, but the point is that the use of a known and popular parametrization, even with limited efficiency, can bring new insights for further developments mainly because it gives information on what should be improved.

\section{CONCLUSIONS AND FINAL REMARKS}

We have obtained novel analytical expressions for the derivative dispersion relations, without high-energy approximations. The mathematical results are valid for the class of functions specified by Theorem 1. In principle, their applicability can be extended to any area that makes use of dispersion techniques, with possible additional constraints, dictated by the analytical and experimental conditions involved. In special, under adequate circumstances, the local character of the derivative operators may be a great advantage.

For scattering amplitudes belonging to the class of functions defined by Theorem 1, the EDDR are valid for any energy above the physical threshold. Since the experimental data on the total cross sections indicate a smooth variation with the energy (without oscillations just above the physical threshold and a smooth systematic increase above $\sqrt{s} \approx 20$ $\mathrm{GeV}$ ), this class includes the majority of functions of physical interest. Using as framework a popular Pomeron-Reggeon parametrization for the total cross sections, we have checked the numerical equivalence between the results obtained with the IDR (finite lower limit $m$ ) and the EDDR, as well as the differences associated with the SDDR and the CMSr. We have also presented a critical discussion on the limitations of the whole analysis from both formal and practical points of view.

We stress that, as in the case of IDR, the practical efficiency of the EDDR in the reproduction of the experimental data on $\sigma_{\text {tot }}$ and $\rho$ depends on the model considered. Here, in order only to check the consistences among the different analytical forms, we made use of a particular Pomeron-Reggeon parametrization, for which a cutoff at $\sqrt{s}=4 \mathrm{GeV}$ was necessary. For example, by considering the full nondegenerated case (four contributions, each one from each meson trajectory, $\left.a_{2}, f_{2}, \rho, \omega\right)$, this cutoff can be reduced [27], or the $\chi^{2} / D O F$ can be reduced for the same cutoff. Despite the limitations of our practical example (Sec. IV), some interesting phenomenological aspects could be inferred. In particular, although already noted [14, 23], we have called the attention to the role of the subtraction constant as a practical "regulator", in the replacement of IDR by derivative forms, a fact that is clearly identified in Table II: the high-energy approximation is absorbed by the constant. In this respect, we have demonstrated that this artifice, which lack physical meaning, can be avoided by the direct use of the EDDR. However, this observation does not depreciate the important role of the subtraction constant as a free fit parameter, since the best statistical results are obtained in this context (Tables I and II). In particular, we note that the effect of this parameter is to provide a slight higher value for the Pomeron intercept, $\alpha_{\mathrm{IP}}(0) \approx 1.088(K=0)$ and $\alpha_{\text {IP }}(0) \approx 1.092$ ( $K$ free).

To our knowledge, a well established theoretical approach for total cross sections just above the physical threshold and in the region connecting low and high energies is still absent. In this sense, despite all the limitations discussed, we hope that the local analytical operators, developed here for these regions, can contribute, as a formal mathematical tool, for further developments on the subject. 


\section{Acknowledgments}

We are thankful to FAPESP for financial support (Contracts No.03/00228-0 and No.04/10619-9).

[1] M.L. Goldberger, Y. Nambu, and R. Oehme, Ann. Phys. 2, 226 (1957); P. Söding, Phys. Lett. 8, 285 (1964).

[2] A. Martin, Nuovo Cimento 42, 930 (1966); 44, 1219 (1966); H. Epstein, V. Glaser, and A. Martin, Comm. Math. Phys. 13, 257 (1969).

[3] M.M. Block and R.N. Cahn, Rev. Mod. Phys. 57, 563 (1985).

[4] N.V. Gribov and A.A. Migdal, Yad. Fiz. 8, 1002 (1968) [Sov. J. Nucl. Phys. 8, 583 (1969)]; J.B. Bronzan, in: Argonne Symposium on the Pomeron, ANL/HEP-7327 (1973) p. 33; J.D. Jackson, in: 1973 Scottish Summer School, LBL-2079 (1973) p. 39.

[5] J.B. Bronzan, G.L. Kane, and U.P. Sukhatme, Phys. Lett. B 49, 272 (1974).

[6] K. Kang and B. Nicolescu, Phys. Rev. D 11, 2461 (1975).

[7] G.K. Eichmann and J. Dronkers, Phys. Lett. B 52, 428 (1974).

[8] J. Heidrich and E. Kazes, Lett. Nuovo Cimento 12, 365 (1975).

[9] G. Höhler, H.P. Jakob, and F. Kaiser, Phys. Lett. B 58, 348 (1975).

[10] A. Bujak and O. Dumbrajs, J. Phys. G: Nucl. Phys. 2, L129 (1976).

[11] I. Vrkoč, Czech. Math. J. 35, 59 (1985); M.J. Menon, A.E. Motter, and B.M. Pimentel, Phys. Lett. B 451207 (1999); Yu. S. Vernov, M.N. Mnatsakanova, Physics of Particles and Nuclei 32, 589 (2001).

[12] J. Fischer and P. Kolár, Phys. Lett. B 64, 45 (1976); Phys. Rev. D 17, 2168 (1978); P. Kolár and J. Fischer, J. Math. Phys. 25, 2538 (1984); J. Fischer and P. Kolář, Czech. J. Phys. B 37, 297 (1987).

[13] P. Kolář and J. Fischer, in Proc. Blois Workshop on Elastic and Diffractive Scattering, Prague, 2002, edited by V. Kundrat and P. Zavada (IOP, Prague, 2002), p. 305.
[14] R.F. Ávila and M.J. Menon, Nucl. Phys. A 744, 249 (2004)

[15] J.R. Cudell, E. Martynov, and O. Selyugin, hep-ph/0307254; E. Martynov J.R. Cudell, and O. Selyugin, Eur. Phys. J. C 33, 533 (2004); hep-ph/0311019.

[16] R.J.M. Covolan, J. Montanha , and K. Goulianos, Phys. Lett. B 389, 176 (1996).

[17] J.R. Cudell, K. Kang, and S.K. Kim, Phys. Lett. B 395, 311 (1997).

[18] S. Eidelman et al., Phys. Lett. B 592, 1 (2004) and 2005 partial update for the 2006 edition available on the PDG www pages (URL http://pdg.lbl.gov).

[19] E811 Collaboration, C. Avila et al., Phys. Lett. B 537, 42 (2002).

[20] CDF Collaboration, F. Abe et al., Phys. Rev. D 50, 5550 (1993).

[21] E710 Collaboration, N.A. Amos et al., Phys. Rev. Lett. 68, 2433 (1992).

[22] E811 Collaboration, C. Avila et al., Phys. Lett. B 445, 419 (1999).

[23] R.F. Ávila, E.G.S. Luna, and M.J. Menon, Phys. Rev. D 67, 054020 (2003).

[24] COMPETE Collaboration, J.R. Cudell et al., Phys. Rev. D 61, 034019 (2000); Phys. Rev. D 63, 0599901(E) (2001).

[25] R.F. Ávila, S.D. Campos, M.J. Menon, and J. Montanha, Eur. Phys. J. C 47, 171 (2006); hep-ph/0603035.

[26] UA4/2 Collaboration, C. Augier et al., Phys. Lett. B 315, 503 (1993).

[27] R.F. Ávila and M.J. Menon, in Sense of Beauty in Physics - A volume in honour of Adriano Di Giacomo, edited by M. D'Elia, K. Konishi, E. Meggiolaro and P. Rossi (Edizioni Plus, Pisa University Press, Pisa, 2006), p. 153; hep-ph/0601194. 